\title{
Deep divergence of Red-crowned Ant Tanager (Habia rubica: Cardinalidae), a multilocus phylogenetic analysis with emphasis in Mesoamerica
}

\author{
Sandra M Ramírez-Barrera ${ }^{1}$ ， Blanca E Hernández-Baños ${ }^{\text {Corresp.., }}{ }^{1}$, Juan P Jaramillo-Correa ${ }^{2}$, John Klicka ${ }^{3}$ \\ 1 Departamento de Biología Evolutiva, Facultad de Ciencias, Museo de Zoología, Universidad Nacional Autónoma de México, Ciudad de México, Mexico \\ 2 Departamento de Ecología Evolutiva, Instituto de Ecología, Universidad Nacional Autónoma de México, Ciudad de México, Mexico \\ 3 Department of Biology and Burke Museum of Natural History and Culture, University of Washington, Seatle, Washington, United States \\ Corresponding Author: Blanca E Hernández-Baños \\ Email address: behb@ciencias.unam.mx
}

Many neotropical species have a complex history of diversification as a result of the influence of geographical, ecological, climatic, and geological factors that determine the distribution of populations within a lineage. Phylogeography identifies such populations, determines their geographic distributions, and quantifies the degree of genetic divergenced. In this work we explored the genetic structure of Habia rubica populations, a polytypic taxon with 17 subspecies describes, in order to obtain hypotheses about their evolutionary history and processes of diversification. We undertook multilocus analyses using sequences of five molecular markers (ND2, ACOI-19, MUSK, FGB-I5 and ODC), and sampling from across the species' distribution range, an area encompassing from Central Mexico throughout much of South America. With these data, we obtained a robust phylogenetic hypothesis, a species delimitation analysis, and estimates of divergence times for these lineages. The phylogenetic hypothesis of concatenated molecular markers shows that Habia rubica can be divided in three main clades, the first includes Mexican Pacific coast populations, the second is formed by population from east of Mexico to Panama and the third comprises the South American populations. Within these clades we recognize seven principal phylogroups whose limits have a clear correspondence with important geographical discontinuities including the Isthmus of Tehuantepec in southern Mexico, the Talamanca Cordillera, and the Isthmus of Panama in North America. In South America, we observed a marked separation of two phylogroups that include the populations that inhabit mesic forests in western and central South America (Amazon Forest) and those inhabiting the seasonal forest from the eastern and northern regions of the continent (Atlantic Forest). These areas are separated by an intervening dry vegetation "diagonal" (Chaco, Cerrado and Caatinga). The geographic and genetic structure of these phylogroups describes a history of diversification more active and complex in the northern 
distribution of this species, producing at least seven well-supported lineages that could be considered species. 
2 Deep divergence of Red-crowned Ant Tanager (Habia rubica: Cardinalidae), a multilocus

3 phylogenetic analysis with emphasis in Mesoamerica

4

5 Sandra M Ramírez-Barrera ${ }^{1}$, Blanca E Hernández-Baños ${ }^{1 *}$, Juan P Jaramillo-Correa ${ }^{2}$ and John

6 Klicka $^{3}$

7

$8{ }^{1}$ Museo de Zoología, Departamento de Biología Evolutiva, Facultad de Ciencias, Universidad

9 Nacional Autónoma de México, Ciudad de México. México.

${ }^{2}$ Departamento de Ecología Evolutiva, Instituto de Ecología, Universidad Nacional Autónoma de

México, Ciudad de México, México.

${ }^{3}$ Department of Biology and Burke Museum of Natural History and Culture, University of

13 Washington, Seattle, Washington, United States of America.

*Corresponding Author: Blanca E. Hernández-Baños

E-mail address: behb@ciencias.unam.mx 
27 ABSTRACT

Many neotropical species have a complex history of diversification as a result of the influence of geographical, ecological, climatic, and geological factors that determine the distribution of populations within a lineage. Phylogeography identifies such populations, determines their geographic distributions, and quantifies the degree of genetic divergenced. In this work we explored the genetic structure of Habia rubica populations, a polytypic taxon with 17 subspecies describes, in order to obtain hypotheses about their evolutionary history and processes of diversification. We undertook multilocus analyses using sequences of five molecular markers (ND2, ACOI-I9, MUSK, FGB-I5 and ODC), and sampling from across the species' distribution range, an area encompassing from Central Mexico throughout much of South America. With these data, we obtained a robust phylogenetic hypothesis, a species delimitation analysis, and estimates of divergence times for these lineages. The phylogenetic hypothesis of concatenated molecular markers shows that Habia rubica can be divided in three main clades, the first includes Mexican Pacific coast populations, the second is formed by population from east of Mexico to Panama and the third comprises the South American populations. Within these clades we recognize seven principal phylogroups whose limits have a clear correspondence with important geographical discontinuities including the Isthmus of Tehuantepec in southern Mexico, the Talamanca Cordillera, and the Isthmus of Panama in North America. In South America, we observed a marked separation of two phylogroups that include the populations that inhabit mesic forests in western and central South America (Amazon Forest) and those 
47 inhabiting the seasonal forest from the eastern and northern regions of the continent (Atlantic

48 Forest). These areas are separated by an intervening dry vegetation "diagonal" (Chaco, Cerrado

49 and Caatinga). The geographic and genetic structure of these phylogroups describes a history of

50 diversification more active and complex in the northern distribution of this species, producing at

51 least seven well-supported lineages that could be considered species.

52

\section{INTRODUCTION}

54 The origins and evolutionary drivers of neotropical diversity are one of the most studied and

55 hotly debated topics since the first biological explorations in the $19^{\text {th }}$ century (Salvin \&

56 Goldman, 1879-1904). Two main hypotheses have been proposed to explain the high levels of

57 neotropical biodiversity (Rull, 2008). On one hand, the glacial-interglacial cycles of the

58 Pleistocene (last 2.6 Mya) have been suggested as the major drivers of divergence, during

59 which allopatric speciation took place in isolated rainforest refuges, particularly during the

60 cooler and drier conditions of the glacial maxima (Whitmore \& Prance, 1987; Hooghiemstra \&

61 van der Hammen, 1998; Hewitt, 2000; Bennett, 2004). On the other hand, the paleogeographic

62 changes that occurred throughout the Cenozoic (i.e. the last $\sim 66$ Mya), like the Andean orogeny

63 and the uprising of the Isthmus of Panama (Hewitt, 2000; Willis \& Niklas, 2004; Nores, 2004),

64 have been postulated as major factors driving diversification, as new geographical barriers both

65 increased isolation and promoted divergence. Both hypotheses have however been criticized. For

66 instance, there is little palynological (Colinvaux et al., 2001; Bush \& de Oliveira, 2006),

67 phylogenetic (e.g. Moritz et al., 2000; Glor, Vitt \& Larson, 2001), or ecological-modeling

68 support (Cowling, Maslin \& Sykes, 2001; Mayle et al., 2004) for a Pleistocene origin of tropical

69 diversity. Indeed, most divergence times estimated so far date back to the Neogene, supporting a 
70 role for more ancient diversification events. However, differentiation patterns across taxa are

71 highly discordant and frequently cannot be linked to specific landscape or vicariant events

72 (Burns \& Naoki, 2004; Burns \& Racicot, 2009; Mauck \& Burns, 2009) as this hypothesis

73 proposes; instead, they seem more related to each species ability to persist through

74 environmental changes and independently disperse across putative geographic barriers (e.g.

75 Smith et al., 2014).

76 The role of ecological divergence in producing neotropical diversity has taken force in

77 the last decade. For instance, alternating cycles of contracting and expanding tropical forests

78 could have resulted in multiple bouts of differentiation that produced assemblages of "old" and

79 "young" taxa exclusive to each habitat (e.g. Pennington et al., 2004; Smith et al., 2013). This is

80 not only important for species diverging in situ, but also for taxa that dispersed and differentiated

81 across newly available habitats, such as those involved in inter-continental exchange after the

82 uplifting of the Isthmus of Panama. It has been suggested that animals tolerant to a variety of

83 habitats and elevation zones quickly expanded through this corridor into North America, while

84 species restricted to more humid conditions could only disperse more recently into northern

85 latitudes (Zamudio-Beltrán \& Hernández-Baños, 2015).

86 Another points that fuels this debate is the species concept itself. That is, evidence

87 supporting one or another hypothesis can be dismissed or not depending on whether the taxa

88 studied are considered (or not) independent species. Traditionally, species boundaries have been

89 drawn based on morphology, but the onset of high-throughput molecular techniques has allowed

90 inferring these boundaries from statistical models based on the coalescent framework (e.g.

91 Bayesian Phylogenetics and Phylogeography; BP\&P; Yang \& Rannala, 2010). As both views

92 have their own limitations (see for instance Sukumaran \& Knowles 2017), an integrative 
93 approach based on different types of data (morphological, ethological, ecological, molecular,

94 etc.) seems necessary to operationally determine whether lineages have been evolving separately

95 and therefore can be considered different species (De Queiroz 2007).

96 The Red-crowned Ant Tanager (Habia rubica, Cardinalidae) is a highly polytypic taxon

97 with marked geographical variation; it comprises up to 17 subspecies (Avibase, 2012) most of

98 which were described based on the variation in the hue and intensity of plumage coloration

99 (Hilty, 2011). Its current distribution ranges from central Mexico to north-eastern Argentina and

100 southeastern Brazil, and encompasses regions with very different ecological conditions and/or

101 separated by recognized biogeographical barriers (Hilty, 2011). A previous phylogeographic

102 survey (Lavinia et al., 2015) suggested that this species originated in South America, where it

103 bears at least two clearly differentiated clades, one in the Atlantic Forest of Brazil and another in

104 the rainforests of the Amazon basin. However, a limited sampling precluded the authors to

105 explore the colonization and diversification within Central America and Southern Mexico.

106 The objective of this paper is to evaluate the nature and geographic structuring of genetic

107 variation within and among populations of Habia rubica, using both nuclear and mitochondrial

108 genetic markers in order to provide a phylogenetic hypothesis about the evolutionary history of

109 the species. We used Bayesian and coalescent methods to generate a phylogeny and estimate

110 divergence times between clades, to assess the validity of the genetic groupings identified using

111 species delimitation methods (Rannala \& Yang, 2003; Yang \& Rannala, 2010). Emphasis was

112 made on the missing parts of the biogeographical history of the species.

113

114 MATERIAL \& METHODS

115 Taxon sampling and laboratory procedures 
116 We sequenced 125 individuals of Habia rubica species that covered the species distribution,

117 including samples from Mexico (Using field collecting permit from Instituto Nacional de

118 Ecología, SEMARNAT: FAUT-0169), Guatemala, El Salvador, Honduras, Nicaragua, Costa

119 Rica, Panama, Peru, Bolivia, Paraguay, Brazil, Argentina and Venezuela (Fig. 1A). We also

120 included 16 samples from the genus Chlorothraupis (C. olivacea, C. carmioli and C.

121 stolzmanni), the sister group of H. rubica (Klicka, Burns \& Spellman, 2007), and three

122 congeneric samples that were used as outgroups (H. fuscicauda, H. atrimaxillaris, and $H$.

123 gutturalis; see Table S1).

124 We isolated genomic DNA from tissues using Qiagen DNAeasy kit (Qiagen, Valencia,

125 CA, USA) following the manufacturer's protocol. We amplified five molecular markers via the

126 polymerase chain reaction (PCR) in $12.5 \mu$ l volumens. The mitochondrial marker ND2 (1041

127 bp), was generated for all individuals $(n=144)$ using the primers L5219 and H6313 (Sorenson et

128 al., 1999). We then amplified four nuclear markers, for a subset of individuals with the highest

129 DNA quality $(n=37)$. These nuclear loci included two Z-linked introns, the aconitase I gene

130 (ACOI-I9, 852 bp) amplified using the primers ACOI-I9F and ACOI-I9R (Kimball et al., 2009),

131 and the receptor of the tyrosine kinase MUSK gene (MUSK, $576 \mathrm{bp}$ ) amplified using the primers

132 MUSK-I3F and MUSK-I3R (Kimball et al., 2009). We also amplified two autosomal loci, a

133 beta-fibrinogen gene ( $F G B$-I5, 577), using the primers Fib5 and Fib6 (Kimball et al., 2009) plus

134 the Ornithine Decarboxylase ODC gene $(O D C, 711 \mathrm{bp})$ using the primers ODCF and ODCR

135 (Primmer et al., 2002). We used the following annealing temperatures: $54^{\circ} \mathrm{C}(\mathrm{ND} 2), 56^{\circ} \mathrm{C}$

136 (MUSK), $60^{\circ} \mathrm{C}$ (ACOI and FGB-I5) and $65^{\circ} \mathrm{C}$ (ODC). PCR success was verified on $1 \%$ agarose 137 gels. 
139 University of Washington. We edited and aligned chromatograms with Sequencher v4.8

140 (GeneCodes Corporation, Ann Arbor, MI, USA). We corroborated the origin of all of our

141 sequences by combining at least 2 of the following methods: amplifying overlapping gene

142 segments, amplifying or sequencing 1 region with different primer sets, sequencing both DNA

143 strands for all amplified fragments, or using multiple individuals of a single species. No evidence

144 of NUMT contamination was found (Zhang \& Hewitt, 1996; Sorenson \& Quinn, 1998;

145 Bensasson et al., 2001), and all nuclear sequences could be easily aligned with the corresponding

146 genes from other avian species. The overall length of the concatenated mitochondrial and nuclear

147 sequences was $\sim 3,800 \mathrm{bp}$. All sequences were deposited in FigShare

148 (https://figshare.com/s/fe9f9f6fed1686782f62).

150 Phylogenetic analyses

151 Phylogenies were constructed from two different matrices; the first matrix included 144

152 sequences of the mitochondrial marker ND2 (hereafter referred to as the mtDNA dataset), and

153 the second matrix included 37 concatenated sequences of the mitochondrial marker ND2 plus the

154 four nuclear markers describe above (hereafter referred to as multilocus dataset). Phylogenetic

155 analyses were performed for each matrix using a Bayesian Inference method (BI), with

156 independent partitions and evolutionary rates defined for each gene region. The model

157 parameters of nucleotide evolution for each DNA region were obtained with jModeltest v3.8

158 (Posada, 2008) using the Akaike Information Criterion (Akaike, 1973). Analyses were conducted

159 in MrBayes v3.2 (Ronquist et al., 2012), and consisted of four MCMC chains of 50 million

160 generations each, starting from random trees and using a uniform prior distribution of 
161 parameters. Trees were sampled every 250 generations, using Tracer v1.6.0 (Rambaut et al.,

162 2013), after discarding the initial $20 \%$ as burn-in. The remaining trees were used to construct a

163 majority rule consensus tree with a posterior probability distribution. The final tree was

164 visualized in FigTree v1.2.3 (http//tree.bio.ed.ac.uk/software/figtre/). Finally, we used MEGA v7

165 (Kumar et al., 2016) to estimate the pairwise genetic distances between lineages from

166 mitochondrial and nuclear markers, applying a bootstrap of 1000 iterations; this allowed us to

167 indirectly determine the relative amounts of information provided by the mitochondrial and

168 nuclear markers.

\section{Diversity and genetic structure}

171 A haplotype network was obtained for the mtDNA dataset with the Median-Joining algorithm 172 available in Network v4.6 (Bandelt, Foster \& Röhl, 1999). We used DnaSP v5.0 (Librado \& 173 Rozas, 2009) for estimating the number of haplotypes $(H)$ and segregating sites $(S)$, the

174 haplotype $(H d)$ and nucleotide $(\pi)$ diversities, and the Tajima's $D$ (Tajima, 1989) and Fu's $F(\mathrm{Fu}$, 175 1997) values for the genetic clusters recovered in the BI mitochondrial phylogeny mentioned 176 above. Significance for the last two summary statistics was inferred from 1000 replicates of the 177 coalescent algorithm.

\section{Species delimitation}

180 The phylogeny obtained from the multilocus dataset was used to assess species delimitations 181 under the Bayesian framework implemented in BP\&P (Yang \& Rannala, 2010). This method 182 allows the estimation of posterior distributions of competing models with differing number of 183 species. It incorporates a reversible-jump Markov chain Monte Carlo (rjMCMC) algorithm and a 
184 user-specified tree, where it assigns speciation probability values (Rannala \& Yang, 2003; Yang

$185 \&$ Rannala, 2010). BP\&P assumes constant population sizes, no gene flow among lineages, and no population structure within lineages after speciation occurred. It furthers includes divergence time $(\tau)$ and mutation-scaled effective population size $(\theta)$ parameters. We first performed several preliminary runs with the two available algorithms (0: fixed tree, and 1: tree as guide tree) and different seed numbers to confirm consistency and fine-tuning the priors ( $\varepsilon$ and $\tau$ ). After selecting algorithm 0 , we conducted a first set of analyses allowing the program to make automatic adjustments to the priors (finetune $=1$ ). Because different values of $\tau$ and $\theta$ can affect the model posterior probabilities for a same guide tree (Leaché \& Fujita, 2010; McKay et al., 2013), and to explore a wide range of speciation histories, we performed a series of simulations using three different parameter combinations: 1$)$ large population size/deep divergence $[\mathrm{G}(1,10)$ for both $\theta$ and $\tau], 2)$ small population size/ shallow divergence $[\mathrm{G}(2,2000)$ for both $\theta$ and $\tau]$, and

3) large population size/shallow divergence $[\mathrm{G}(1,10)$ for $\theta$ and $G(2,2000)$ for $\tau]$ (Leaché \& Fujita, 2010; Smith et al., 2013). We then performed a final set of analyses that incorporated the finetune parameters determined in the previous analysis (finetune $=0$ ) with the same three different combinations for $\theta$ and $\tau$ parameters described above. Each set was composed of 100,000 generations, and samples were taken every 5 steps after discarding the initial 50,000 iterations as burn-in. All analyses were performed on the BPPX graphics user interface (http://abacus.gene.ucl.ac.uk/software) using the command line.

\section{Tests of divergence times}

Divergence times were inferred from the multilocus dataset. Analyses were performed in Beast 
207 corresponding evolutionary rates and models as estimated above (Ellegren, 2007; Smith \&

208 Klicka, 2010) and the seven phylogroups identified with the multilocus dataset. Clade divergence

209 was simulated as a Yule's first tree (Yule, 1924) with a lognormal relaxed molecular clock

210 (Drummond et al., 2006). Analyses included 100 million iterations, with samples taken every

2111,000 generations after eliminating $25 \%$ of the trees as burn-in. Mixing and likelihood stability

212 were confirmed with TRACER v1.6 (Rambaut \& Drummond, 2013) to make sure that the

213 appropriate effective sample size (ESS) was over 200 units for each parameter (Drummond \&

214 Rambaut, 2007). The best supported tree was chosen with TreeAnnotator 1.8.0 (Drummond \&

215 Rambaut, 2007) through Maximum Clade Credibility.

Given the absence of fossils for H. rubica, or any close relatives, two calibrations based

217

218

219

220

221

222

223

224

225

226

227

228

229

on geological data were used. First, the closing of the Isthmus of Panama, estimated between 3.1

and 4.0 Ma (Daza, Castoe \& Parkinson, 2010; Smith \& Klicka, 2010), was used for the

separation of South and Central American populations; and second, the uprising of the

Talamanca Cordillera near the Costa Rica - Panama border, estimated to have occurred between

2.5 to 3.9 Ma (Marshall \& Liebherr, 2000; Daza, Castoe \& Parkinson, 2010), was employed as

the time of divergence between populations in Panama and those occurring elsewhere throughout

Mesoamerica. In addition, as estimating divergence times based on external event that could be

unrelated to a species biology can result in wrong inferences and circular reasoning, we

estimated divergence dates a second time but using only the mtDNA dataset (which has a higher sample size) and no calibration points. This could be seen as an independent test of confidence

for the estimates obtained for the multilocus analysis with geological constraints. We assumed a mutation rate of $1.25 \times 10^{-2}$ sustitutions/site/Ma (Smith \& Klicka, 2010), other parameters and priors were kept identical. 


\section{RESULTS}

232

233

234

235

236

237

238

239

240

241

242

243

244

245

246

247

248

249

250

251

252

\section{Phylogenetic analyses}

We obtained mitochondrial (1041 bp) and concatenated (3,757 bp) datasets. The best-fit models for each molecular markers were follows: ND2, TrN+I+G; ACOI-I9, TIM2+G; MUSK, TrN+G; FGB-I5, HKY+G and ODC, TrN.

The phylogenies obtained with mitochondrial and multilocus dataset revealed wellsupported topologies, independently of the method employed. Both topologies are composed of three main clades with seven and eight subclades (considered here as "phylogroups") for the multilocus and mitochondrial datasets, respectively. . The first clade in the multilocus phylogeny (I) is comprised of two phylogroups with an apparent separation between populations occupying the western (A; Fig. 1B) and southern (B; Fig. 1B) Pacific coast of Mexico. The second clade (II) was composed of three well-supported phylogroups distributed from eastern Mexico to Panama. The first includes all individuals from the Mexican Gulf Coast, west of the Isthmus of Tehuantepec (C; Fig. 1B); the second one comprised samples collected east of this Isthmus and southward into Nicaragua (D; Fig. 1B); and the third one was composed by samples from Panama (E; Fig. 1B). The last clade (III) was composed of (at least) two phylogroups, one with individuals from the Amazon basin (F; Fig. 1B) and another one with birds from eastern South America (G; Fig. 1B). A single sample from Venezuela appears distinctive and is sister to this eastern clade. However, additional samples from Venezuela will be required for a proper systematic assessment.

The mitochondrial phylogeny, although based on many more samples, was almost identical to the one obtained with the multilocus dataset (Fig. S1); it did however, show that 
253 phylogroup D was composed of two subgroups: one comprised of individuals from the Yucatan

254 Peninsula, Honduras, Nicaragua and Costa Rica, and the other one of individuals from

255 Guatemala and El Salvador.

256 The pairwise genetic distances showed significant genetic differentiation in the

257 mitochondrial but not in the nuclear markers (Table 1). That is, the genetic distances were

258 smaller for nuclear than for mitochondrial markers (Table 1). This implies that the mitochondrial

259 marker provides larger amounts of relative information than the nuclear gene-regions (Table 2).

261 Diversity and Genetic structure

262 The haplotype mtDNA network (Fig. 1C) revealed the same eight groups obtained with BI

263 phylogeny (Fig. S1). A high number of haplotypes had to be reconstructed to link some of these

264 groups. For instance, 30 mutational steps separated the two groups from the Mexican Pacific

265 coast from the other clusters, 45 more steps were needed to link the clades from South and

266 Central America, while the two groups from South America were 41 steps apart; an additional 29

267 mutations were necessary to join the lone sample from Venezuela to the southeastern South

268 America clade.

269

Overall, diversity estimates differed greatly between mtDNA phylogroups, with the South

American and Panamanian ones being the more diverse, and the groups from the Mexican

271 Pacific coast the least genetically rich. Tajimas $D$ and Fu's $F$ values were negative in all

272 phylogroups, excepting phylogroup D (eastern Mexico to northern Central America). However,

273 these values were significant only for the two South American Clades (F \& G); thus suggesting

274 recent population expansions (Table S2). 


\section{Species delimitation}

277 Considering a hypothesis of seven species for this taxon, the Bayesian species delimitation

278 supported the guide tree displaying speciation probabilities of 1.0 in most nodes, independently

279 of the finetune parameter used (finetune $=1$ or 0 , Fig. $2 \mathrm{~A}$ and $2 \mathrm{~B}$ respectively). Seven species

280 were supported with high speciation probabilities $(0.97$ to 1.0$)$ on the tree guide. These species

281 corresponded to the lineages A, B, C, D, E, F and G. The different prior distributions for $\theta$ and

$282 \tau$ had no effect on most nodes, the only exception being that of lineages A and B which were

283 influenced by at least one of the prior combinations (from 0.37 to 0.97 ). However, while these

284 and other analyses with similar topologies and highly supported clades suggest a multi-species

285 complex within $H$. rubica, other types of data are still necessary to support this hypothesis

286 (Sukumaran \& Knowles, 2017; see below).

287

288

Tests of divergence times

289

290

291

292

293

294

295

296

297

298
According the multilocus dataset, the divergence between Habia rubica and Chlorothraupis dated back to the late Neogene, between 6.65 and 3.91 Myr ago (Fig. 3A), and the ensuing diversification of $H$. rubica was rather rapid. The first split was estimated some 3.75 to 3.35 Myr, between the South American (clade III) and Central/Mesoamerican lineages (clades I and II); these two groups split again around $3 \mathrm{Myr}$ ago. In Central America, the first divergence event (3.4 - 2.7 Myr) occurred between the lineages from the Mexican Pacific coast (Clade I) and the other phylogroups (Clade II). Within clade II, there was a gradual and northward divergence of lineages from Panama to eastern Mexico; these three diversification events during the last $\sim 3 \mathrm{Myr}$ or $\sim 2 \mathrm{Myr}$. An independent analysis performed on the mtDNA dataset and with no calibration constraints, produced an identical topology with older mean divergence times (i.e. divergence 
299

300

301

302

303

304

305

306

307

308

309

310

311

312

313

314

315

316

317

318

319

320

within $H$. rubica occurred during the last 5.6 Myr). However, as confidence intervals were much wider (Fig. 3B) and overlapped with those obtained with the multilocus dataset, they provide support for the dates estimated based on geological times.

\section{DISCUSSION}

Our results show that Habia rubica has considerable geographic genetic structure. We found seven lineages through its distribution; five of them are in Mesoamerica, where the evolutionary history has been more dynamic and complex, and two in South America (this last results is similar with was found by Lavinia et al., 2015). Most lineages are delimited by recognized geographic and ecological barriers, like the Rio Balsas depression in Mexico, the Central American Volcanic Arc, the Isthmus of Panama in Central America, or the dry vegetation diagonal in South America. We recovered a reciprocally monophyletic relationship between $H$. rubica and the genus Chlorothraupis, which coincides with previous studies (Klicka, Burns \& Spellman 2007; Klicka, Johnson \& Lanyon, 2000; Barker et al., 2015; Lavinia et al., 2015); therefore the genus Habia must be considered paraphyletic.

\section{Phylogeny, Diversity and Genetic structure}

The mitochondrial DNA topology indicates that populations of Mexico, Central and South America are profoundly differentiated. In this topology eight phylogroups were identified, six in Mesomerica and two in South America (Fig. S1). The relationship between mitochondrial haplotypes could be determined by the action of various historical processes that promoted a deep genetic structure in Mesoamerica, resulting in a clear example of cryptic speciation. This 
321 complicated phylogenetic structure in Mesoamerica is consistent with the complex geological

322 and biogeographic history of the region (Coates \& Obando, 1996), and the emergence of

323 geographic features such as the Rio Balsas depression, the Isthmus of Tehuantepec in southern

324 Mexico, the Motagua-Polochic-Jocotán fault system in the North of Central America, and the

325 Talamanca Cordillera in Costa Rica, which presumably played roles in shaping the observed

326 geographic and genetic patterns (Daza, Castoe \& Parkinson, 2010; Gutiérrez-García \& Vázquez-

327 Domínguez, 2012, 2013; Suárez-Atilano, Burbrink \& Vázquez-Domínguez, 2014). Our results

328 together with the study of Lavinia et al. (2015) provide a robust hypothesis of the evolutionary

329 history of Habia rubica. For instance, our study confirms the main results of Lavinia et al.

330 (2015) for South America, and improves the resolution for Mesoamerica given our larger sample

331 size and wider area covered.

In South America, our results, in combination with those of Lavinia et al. (2015), showed

333

334

335

336

337

338

339

340

341

342

343

two distinct phylogroups that reflected population divergence according to the habitats preferred

by Habia rubica (Pennington et al., 2004, 2009; Lavin, 2006; Smith et al., 2013, 2014;

Pennington \& Lavin, 2016): one western phylogroup distributed in the mesic forest of the

Amazon basin, and another one in the seasonal forests of the eastern and northern parts of the

continent. This last lineage included the single sample from Venezuela, instead of the western

clade that is geographically more proximate (Prates et al., 2016; Prates et al., 2017). This could

be indicating that the deep evolutionary history of the seasonal forests in South America

(Pennington et al., 2004, 2009) are driving the divergence of other taxa. Additional sampling in this region is thus necessary to test this hypothesis.

The multilocus DNA topology identified seven of the eight phylogroups found with the mitochondrial DNA (Fig. 1B), in all cases the phylogroups are the same with the exception of an 
344 additional separation of a Yucatan Peninsula and northern of Central America clade identified

345 using the more rapidly evolving mtDNA marker. All identified phylogroups (mitochondrial and

346 multilocus dataset) are consistent with clear geographical discontinuities and are supported with

347 high posterior probabilities.

348 Although, the haplotype network recovered the same groups identified in the

349 mitochondrial DNA phylogeny (Fig. 1C), it was necessary to invoke five hypothetical haplotypes

350 between Mexican and Central American groups. These results support the idea about the

351 complex geological history in Mesoamerica resulting in a high degree of genetic structure in the

352 northernmost distribution of this species. In South America, 12 hypothetical haplotypes were

353 required, but unlike the previous case, this could be explained by the lack of sampling in this

354 specific area.

The genetic distances between phylogroups were relatively high and supported the groups

356

357

358

359

360

361

362

363

364

365 identified in our phylogenetic and BPP analyses. The largest difference found was between the group from the Western Pacific Coast of Mexico (group A) and the group from the Southeast of

South America (group G) (Table 1). This result is not surprising because the geographical distance between them is also the largest and is congruent with the divergence date (Fig. 3A and 3B). On the other hand, the more similar phylogroups were the Western (A) and the Southern Pacific Coast of Mexco (B) groups, which represent the most recent divergence event (Fig. 3A and 3B). As expected the mitochondrial DNA provides more poymorphic sites because its rate of evolution is faster (Table 2) (Brown, George \& Wilson, 1979).

\section{Species delimitation and Divergence times}


366 The deep divergence times among lineages estimated herein (Fig. 3A and 3B), most of which

367 dated back to Pleistocene and Pliocene, support the criticisms raised regarding the allopatric

368 speciation hypothesis that invoked the Pleistocene glacial cycles as the major drivers of

369 neotropical bird diversity and closing of the Isthmus of Panama (e.g. Moritz et al., 2000;

370 Cowling, Maslin \& Sykes, 2001; Glor, Vitt \& Larson, 2001; Mayle et al., 2004). According to

371 the ND2 divergence analyses, the first split was during the Miocene (5.69 Myr), and this is the

372 main discordance with respect to the calibrated multilocus divergence analyses (3.55 Myr).

373 These dates are also consistent with the compelling evidence for speciation drawn from the

374 BP\&P analyses, which suggest that enough differences have accumulated between most of the

375 Habia rubica lineages to consider them a complex of cryptic species (Fig. 2).

376 At present, intraspecific delimitation within H. rubica is based on geographic variation in

377 plumage color, with 17 subspecies currently recognized (Avibase, 2012). Many of these

378 subspecies are not supported by the lineages described herein (Fig. 2). For instance, the

379 subspecies rosea and rubicoides are paraphyletic (lineages A\&B, and C\&D, from BP\&P

analysis, respectively). The first taxon is further intermingled with ssp. affinis individuals in

381 lineage $\mathrm{B}$, while the second one is combined with individuals of ssp. holobrunnea and nelsoni in

the lineages $\mathrm{C}$ and $\mathrm{D}$. Some subspecies also appear indistinguishable with the markers that we used (e.g. ssp. hesterna, peruviana and rhodinolaema within lineage F). Overall, our results support a taxonomic revision of $H$. rubica.

Our test for species delimitation suggests that Habia rubica should minimally be split 386 into the following species: 1 . H. rosea, distributed along the Pacific coast of western Mexico (Jalisco, Nayarit and Colima; lineage A); 2. H. affinis, distributed along the Pacific coast of 
over the slope of Gulf of Mexico (lineage C); 4.- H. rubicoides, distributed from eastern Mexico

390

391

392

393

394

395

396

397

398

399

400

401

402

403

404

405

406

407

408

409

410

\section{CONCLUSIONS}

to southern Nicaragua (lineage D); 5. H. vinacea, allopatric in Panama (lineage E); 6. H.

rhodinolaema, distributed in the Amazon basin (lineage F); and 7. H. rubica, distributed in eastern Brazil, Argentina, and Paraguay (lineage G). Further tests and sampling should be necessary for a more adequate description of the Venezuela's individuals, currently classified as spp. perijana (Hilty, 2011).

There are criticisms to the BP\&P analysis implemented herein. For instance, simulation data have shown that it tends to misidentify population structure as species boundaries (Sukurmaran \& Knowles 2017). However, it must be noted that our delimitation not only detected genetics lineages, but lineages whose distribution coincides with the geographical barriers and ecological differences (habitat preference) of each taxon. That is, it highlights the role of other factors, like vicariance, dispersal and ecology, under a framework of ecological allopatric speciation, which is one the drivers proposed for generating bird species diversity in the neotropics (Hooghiemstra \& van der Hammen, 1998; Hewitt, 2000; Bennett, 2004; Smith et al., 2013). It also shows that this bird diversity is underestimated, and that species cannot be described or estimated based only on morphological criteria. From this point of view, diversifying the techniques for species delimitation, like incorporating bioacoustics, highthroughput sequencing, niche modelling and advanced statistical tools, will also help addressing consequential questions in taxonomy and biogeography. For instance, such multifactorial data should help determining operationally whether the $H$. rubica lineages inferred herein are indeed evolving separately (see De Queiroz, 2007). 
412 The phyogenies obtained independently with mitochondrial and multilocus datasets revealed 413 well-supported topologies for the polytypic taxon Habia rubica. Both topologies are composed 414 of three main clades with eight and seven phylogroups (lineages), respectively. The mtDNA 415 haplotype network produced the same eight groups obtained with the mitochondrial data. The 416 Bayesian species delimitation analysis found seven species highly supported: 1) H. rosea, 417 distributed along the Pacific coast of western Mexico, 2) H. affinis, distributed along the Pacific 418 coast of southern Mexico, 3) H. holobrunnea, distributed distributed over the slope of Gulf of 419 Mexico, 4) H. rubicoides, distributed from eastern Mexico to southern Nicaragua, 5) H. vinacea, 420 allopatric in Panama, 6) H. rhodinolaema, distributed in the Amazon basin and 7) H. rubica, 421 distributed in eastern Brazil, Argentina and Paraguay. Further tests and sampling should be 422 necessary for a more adequate description of the samples from Venezuela, currently classified as spp. perijana. The species subdivision of H. rubica coincides with geographical barriers and ecological differences between taxa, highlighting the role of vicariance, dispersal and ecology in bird species diversification in the neotropics.

\section{ACKNOWLEDGMENTS}

We thank the following institutions for providing samples: Museo de Zoología "Alfonso L. Herrera (UNAM)", The Burke Museum (UWBM), El Colegio de la Frontera Sur (ECOSUR-Ch), the Louisiana Museum of Natural History (Louisiana State University) and the Natural History Museum (University of Kansas). We are also grateful to Isabel Vargas Fernández, Raul Ivan Martínez, Luz Estela Zamudio Beltrán, Ernesto Espinosa Jaramillo and Alejandro Gordillo Martínez for technical help. 
435

436

437

438

439

440

441

442

443

444

445

446

447

448

449

450

451

452

453

454

455

456

\section{REFERENCES}

Akaike H. 1973. Information theory and an extension of the maximum likelihood principle. Second international symposium on information theory. Akademinai Kiado, 267-281.

Avibase. 2012. The world bird database: factsheet of Habia rubica. Available from: https://avibase.bsc-eoc.org/avibase.jsp?lang=EN (accessed 13 February 2018).

Barker FK, Burns KJ, Klicka J, Lanyon SM, Lovette IJ. 2015. New insights into New World biogeography: An integrated view from the phylogeny of blackbirds, cardinals, sparrows, tanagers, warblers, and allies. The Auk 132:333-348.

Bandelt HJ, Foster P, Röhl A. 1999. Median-Joining networks for inferring intraspecific phylogenies. Molecular Biology and Evolution 16:37-48.

Bennett KD. 2004. Continuing the debate on the role of Quaternary environmental change of macroevolution. Philosophical Transactions of the Royal Society, Series B: Biological Sciences 359:295-303.

Bensasson D, Petrov DA, Zhang DX, Hartl DL, Hewitt GM. 2001. Genomic gigantism: DNA loss is slow in mountain grasshoppers. Molecular Biology and Evolution 18:246-253.

Brown WM, George MJr, Wilson AC. 1979. Rapid evolution of animal mitochondrial DNA. Proceedings of National Academy of Sciences of the United States of America 76:19671971.

Burns KJ, Naoki K. 2004. Molecular phylogenetics and biogeography of Neotropical tanagers in the genus Tangara. Molecular Phylogenetic and Evolution 32:838-854.

Burns KJ, Racicot RA 2009. Molecular phylogenetics of a clade of lowland tanagers: implications for avian participation in the Great Interchange. The Auk 126:635-648 
457 Bush MB, de Oliveira, PE. 2006. The rise and fall of the refugial hypothesis of Amazonian 458 speciation: a paleoecological perspective. Biota Neotropica 6:1-17.

459 Coates AG, Obando, JA 1996. The geologic evolution of the Central American isthmus. In:

460 Jackson, J.B.C., Ludd, A.F., Coates, A.G. (Eds.). Evolution and environment in tropical 461 America. University of Chicago Press, Chicago, 21-56.

462 Colinvaux P.A, Irion G, Räsänen ME, Bush MB, Nunes de Mello JAS. 2001. A paradigm to 463 be discarded: geological and paleoecological data falsify the HAFFER and PRANCE refuge hypothesis of Amazonian speciation. Amazoniana 16:609-646.

Cowling SA, Maslin MA, Sykes M. 2001. Paleovegetation simulations of lowland Amazonia and implications for Neotropical allopatry and speciation. Quaternary Research 55:140149.

Daza JM, Castoe TA, Parkinson CL. 2010. Using regional comparative phylogenetic data from snake lineages to infer historical processes in Middle America. Ecography 33:343354.

De Queiroz K. 2007. Species concepts and species delimitation. Systematic Biology. 56:879886.

Drummond AJ, Ho SY, Phillips MJ, Rambaut A. 2006. Relaxed phylogenetics and dating with confidence. PLoS Biol 4, e88.

Drummond AJ, Rambaut A. 2007. BEAST: Bayesian evolutionary analysis by sampling trees. BMC Evolutionary Biology 7:214.

Drummond AJ, Suchard MA, Xie D, Rambaut A. 2013. Bayesian phylogenetics with BEAUti and the BEAST 1.7. Molecular Biology and Evolution 29:1969-1973. 
479

480

481

482

483

484

485

486

487

488

489

490

491

492

493

494

495

496

497

498

499

500

Ellegren, H. 2007. Molecular evolutionary genomics of birds. Cytogenetics and Genome Research 117:120-130.

Fu YX. 1997. Statistical test of neutrality of mutation against population growth, hitchhiking and background selection. Genetics 147:915-925.

Glor RE, Vitt LJ, Larson A. 2001. A molecular phylogenetic analysis of diversification in Amazonian Anolis lizards. Molecular Ecology 10:2661-2668.

Gutiérrez-García TA, Vázquez-Domínguez E. 2012. Biogeographically dynamic genetic structure bridging two continents in the monotypic Central American rodent Ototylomys phyllotis. Biological Journal of the Linnean Society 107:593-610.

Gutiérrez-García TA, Vázquez-Domínguez E. 2013. Consensus between genes and stones in the biogeographic and evolutionary history of Central America. Quaternary Research 79:311-324.

Hewitt G. 2000. The genetic legacy of the Quaternary ice ages. Nature 405, 907-913.

Hilty, SL. 2011. Family Thraupidae (Tanagers) In: del Hoyo J, Elliott A, Christie DA (Eds.), Handbook of the Birds of the World. Vol. 16. Tanagers to New World Blackbirds. Lynx Editions, Barcelona. 46-329.

Hooghiemstra H, van der Hammen T. 1998. Neogene and Quaternary development of the neotropical rain forest: the forest refugia hypothesis, and a literature overview. EarthScience Reviews 44:147-183.

Kimball RT, Braun EL, Barker FK, Bowie RCK, Braun MJ, Chojnowski JL, Hackett SJ, Han KL, Harshman J, Heimer-Torres V, Holznagel W, Huddlestone CJ, Marks BD, Miglia KJ, Moore WS, Reddy S, Sheldon FH, Smith JV, Witt CC, Yuri T. 2009. A 
501

502

503

504

505

506

507

508

509

510

511

512

513

514

515

516

517

518

519

520

521

well-tested set of primers to amplify regions spread across the avian genome. Molecular Phylogenetics and Evolution 50:654-660.

Klicka J, Johnson KP, Lanyon SM. 2000. New World nine-primaried oscine relationships: constructing a mitochondrial DNA framework. The Auk 117:321-336.

Klicka J, Burns K, Spellman GM. 2007. Defining a monophyletic Cardinalini: a molecular perspective. Molecular Phylogenetics and Evolution 45:1014-1032.

Kumar S, Stecher G, K Tamura. 2016. MEGA7: Molecular Evolutionary Genetics Analysis version 7.0 for datasets. Molecular Biology and Evolucion 33: 1870-1874.

Lavin M. 2006. Floristic and geographic stability of discontinuous seasonally dry tropical forests explains patterns of plant phylogeny and endemism, Chapter 19. 433-447 In: Pennington, R. T., Ratter, J. A., Lewis, G. P (Eds.), Neotropical savannas and seasonally dry forests: plant biodiversity, biogeographic patterns and conservation. CRC Press, Boca Raton, Florida.

Lavinia PD, Escalante P, García NC, Barreira AS, Trujillo-Arias N, Tubaro PL, Naoki K, Miyaki CY, Santos FR, Lijtmaer DA. 2015. Continental-scale analysis reveals Deep diversification within the polytypic Red-crowned Ant Tanager (Habia rubica, Cardinalidae). Molecular Phylogenetics and Evolution 89:182-193.

Leaché AD, Fujita MK. 2010. Bayesian species delimitation in West African forest geckos (Hemidactylus fasciatus). Proceedings of the Royal Society 277:3071-3077

Librado P, Rozas J. 2009. DnaSP v5: A software for comprehensive analysis of DNA polymorphism data. Bioinformatics 25:1451-1452. 
522 Mauck WM, Burns KJ. 2009. Phylogeny, biogeography, and recurrent evolution of divergent

523 bill types in the nectar-stealing flowerpiercers (Thraupini: Diglossa and Diglossopis). Biological Journal of the Linnean Society 98:14-28.

525

526

527

528

529

530

531

532

533

534

535

536

537

538

539

540

541

542

543

544

Marshall, C.J, Liebherr JK. 2000. Cladistic biogeography of Mexican transition zone. Journal of Biogeography 27:203-216.

Mayle FE, Beerling DJ, Gosling WD, Bush MB. 2004. Responses of Amazonian ecosystems to climatic and atmospheric carbon dioxide changes since the last glacial maximum. Philosophical Transactions of the Royal Society of London B 359:499-514.

McKay BD, Mays HL Jr, Wu Y, Li H, Yao CT, Nishiumi I, Zou F. 2013. An empirical comparison of character-based and coalescent-based approaches to species delimitation in a young avian complex. Molecular Ecology 22:4943-4957.

Moritz C, Patton JL, Schneider CJ, Smith BT. 2000. Diversification of rainforest faunas: An integrated molecular approach. Annual Review of Ecology and Systematics 31:533-563.

Nores M. 2004. The implications of Tertiary and Quaternary sea level rise events for avian distribution patterns in the lowlands of northern South America. Global Ecology and Biogeography 13:149-161.

Pennington RT, Lavin M, Prado DE, Pendry CA, Pell SK, Butterworth CA. 2004. Historical climate change and speciation: neotropical seasonally dry forest plants show patterns of both Tertiary and Quaternary diversification. Philosophical Transactions of the Royal Society of London B 359:515-537.

Pennington RT, Lavin M, Oliveira-Filho A. 2009. Woody plant diversity, evolution, and ecology in the tropics: perspectives from seasonally dry tropical forests. Annual Review of Ecology, Evolution and Systematics 40:437-457. 
545 Pennington RT, Lavin M. 2016. The contrasting nature of woody plant species in different

546 neotropical forest biomes reflects differences in ecological stability. The New Phytologist 210:25-37.

548

549

550

551

552

553

554

555

556

557

558

559

560

561

562

563

564

565

566

567

Posada D. 2008. jModelTest: Phylogenetic Model Averaging. Molecular Biology and Evolution 25:1253-1256.

Prates I, Rivera D, Rodrigues MT, Carnaval AC. 2016. A mid-Pleistocene rainforest corridor enabled synchronous invasions of the Atlantic Forest by Amazonia anole lizards. Molecular Ecology 25: 5174-5186.

Prates I, Melo-Sampaio PR, de Oliveira L, Teixeira MJr, Rodrigues MT, Carnaval AC. 2017. Biogeographic links between southern Atlantic Forest and western South America: Rediscovery, re-description, and phylogenetic relationships of two rare montane anole lizards from Brazil. Molecular Phylogenetics and Evolution 113: 49-58.

Primmer CR, Borge T, Lindell J, Saetre GP. 2002. Single-nucleotide polymorphism characterization in species with limited available sequence information: high nucleotide diversity revealed in the avian genome. Molecular Ecology 11:603-612.

Rambaut, A, Drummond, A. 2013. Tracer v16.0. Available at: http://beast.bio.ed.ac.uk/Tracer

Rannala B, Yang Z. 2003. Bayes estimation of species divergence times and ancestral population sizes using DNA sequences from multiple loci. Genetics 164:1645-1656.

Ronquist F, Teslenko M, Van der Mark P, Ayres DL, Darling A, Höhna B, Liu L, Suchard MA, Huelsenbeck JP. 2012. MrBayes 3.2: efficient Bayesian inference and model choice across a large model space. Systematic Biology 61:539-542.

Rull V. 2008. Speciation timing and neotropical biodiversity: The Tertiary-Quaternary debate in the light of molecular phylogenetic evidence. Molecular Ecology 17:2722-2729. 
568 Salvin O, Godman FD. 1879-1904. Biologia Centrali-Americana, Aves. Taylor and Francis, $569 \quad$ London. 4 vols.

570 Smith BT, Klicka J. 2010. The profound influence of the Late Pliocene Panamanian uplift on 571 the exchange, diversification, and distribution of New World birds. Ecography 33:333$572 \quad 342$.

573 Smith BT, Ribas CC, Whitney BM, Hernández-Baños BE, Klicka J. 2013. Identifying biases 574 at different spatial and temporal scales of diversification: a case study in the Neotropical parrotlet genus Forpus. Molecular Ecology 22:483-494.

576

577

578

579

580

581

582

583

584

585

586

587

588

589

590

Smith BT, McCormack JE, Cuervo AM, Hickerson MJ, Aleixo A, Cadena CD, PérezEmán J, Burney CW, Xie X, Harvey MG, Faircloth BC, Glenn TC, Derryberry EP, Prejean J, Fields S, Brumfield RT. 2014. The drivers of tropical speciation. Nature 515:406-409.

Sorenson MD, Quinn TW. 1998. Numts: A challenge for avian systematics and population biology. The Auk 115:214-221.

Sorenson MD, Ast CJ, Dimcheff DE, Yuri T, Mindell DP. 1999. Primers for PCR-based approach to mitochondrial genome sequencing in birds and other vertebrates. Molecular Phylogenetics and Evolution 12:105-114.

Suárez-Atilano MA, Burbrink F, Vázquez-Domínguez E. 2014. Phylogeographical structure within Boa constrictor imperator across the lowlands and mountains of Central America and Mexico. Journal of Biogeography 41:2371-2384.

Sukumaran J, Knowles LL. 2017. Multispecies coalescent delimits structure, not species. Proceedings of the National Academy of Sciences of the United States of America. 114:1607-1612. 
591 Tajima F . 1989. Statistical method for testing the neutral mutation hypothesis by DNA poly$592 \quad$ morphism. Genetics 123:585-595.

593 Whitmore TC, Prance GT. 1987. Biogeography and Quaternary history in tropical America. $594 \quad$ (Eds). Clarendon Press, Oxford.

595 Willis KJ, Niklas KJ. 2004. The role of Quaternary environmental change in plant 596 macroevolution, the exception or the rule? Philosophical Transactions of the Royal Society, Series B: Biological Sciences 359:159-172.

598

599

600

601

602

603

604

605

606

Yang Z, Rannala B. 2010. Bayesian species delimitation using multilocus sequence data. Proceedings of the National Academy of Sciences USA 107:9264-9269.

Yule GU. 1924. A mathematical theory of evolution: based on the conclusions of Dr. J. C. Willis. Philosophical Transactions of the Royal Society of London B 213:21-87.

Zamudio-Beltrán LE, Hernández-Baños BE. 2015. A multilocus analysis provides evidence for more than one species within Eugenes fulgens (Aves: Trochilidae). Molecular Phylogenetics and Evolution 90:80-84.

Zhang DX, Hewitt GM. 1996. Nuclear integrations: challenges for mitochondrial DNA markers. Trends in Ecology and Evolution 11:247-251. 


\section{Figure 1}

Geographical distribution, phylogenetic consensus tree and haplotypes network

(A) Geographical distribution (indicated by pink shading) and sampling points of the Habia rubica species; the mitochondrial DNA sampling is represented by dots color and the nuclear DNA sampling is highlighted with a black dot on the dots color. ArcGIS (ArcMAP 10.2.2). (B) Phylogenetic consensus tree representing the relationship among populations from $\mathrm{H}$. rubica, based on Bayesian inference from a multilocus dataset. Values above branches denote posterior probabilities (PP). (C) Haplotypes network, where the phylogroup " $\mathrm{D}$ " corresponds with individuals from Chiapas-Yucatan peninsula to Costa Rica and " $d$ " corresponds with individuals from Guatemala and El Salvador (the number inside of circles indicated the number of individuals that shared each haplotype).

A

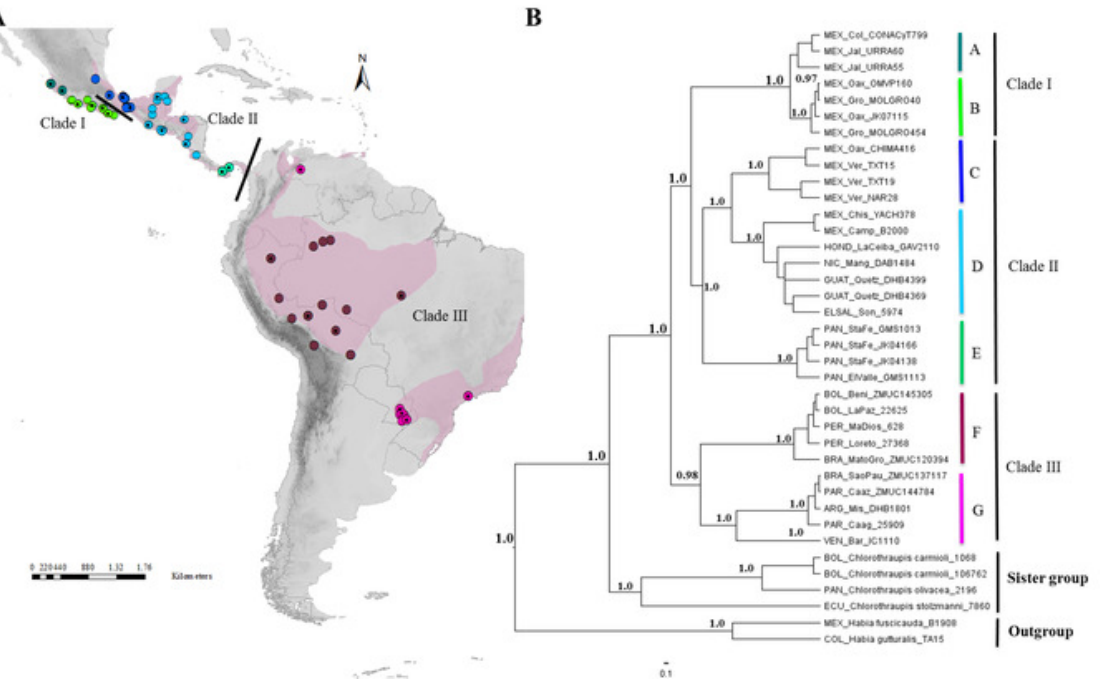

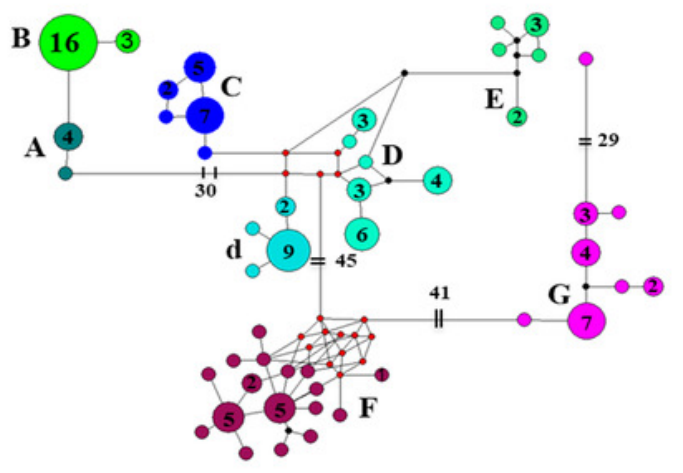


Figure 2

Bayesian species delimitation

(A) Bayesian species delimitation results assuming seven species (lineages) from multilocus phylogeny of Habia rubica (Finetune $=1$ ). (B) Bayesian species delimitation results assuming seven species (lineages) from multilocus phylogeny of Habia rubica (Finetune $=0$, parameters estimated previously). The speciation probabilities are provided for each node under each combination of priors for $\tau$ and $\theta$ : top, priors $\theta \sim G(1,10)$ and $\tau 0 \sim G(1,10)$; middle, priors $\theta \sim G(2,2000)$ and $\tau 0 \sim G(2,2000)$; bottom, priors $\theta \sim G(1,10)$ and $\tau 0 \sim G(2,2000)$. We consider speciation probability values $>0.95$ as strong support for a speciation event.

*Note: Auto Gamma Correction was used for the image. This only affects the reviewing manuscript. See original source image if needed for review.

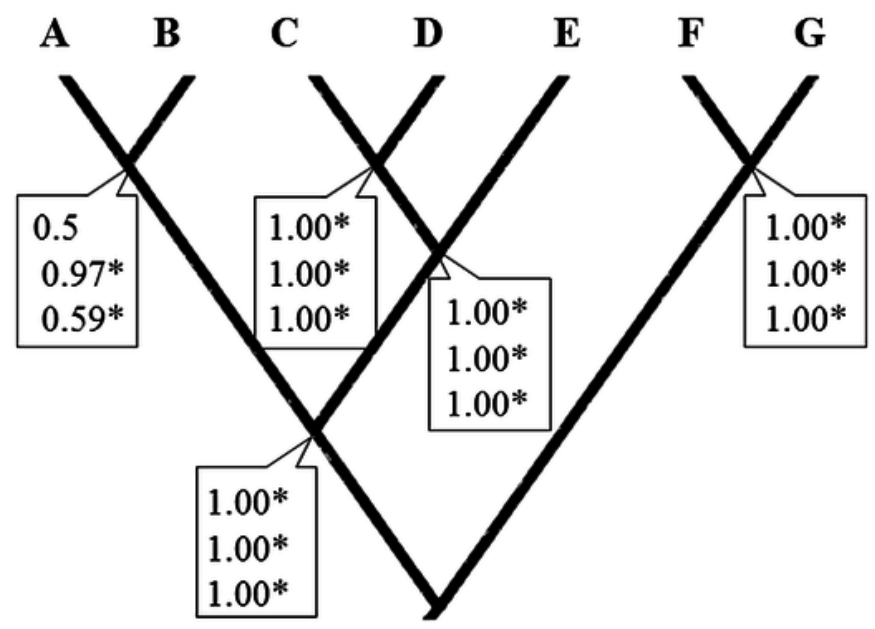

$\mathbf{A}$

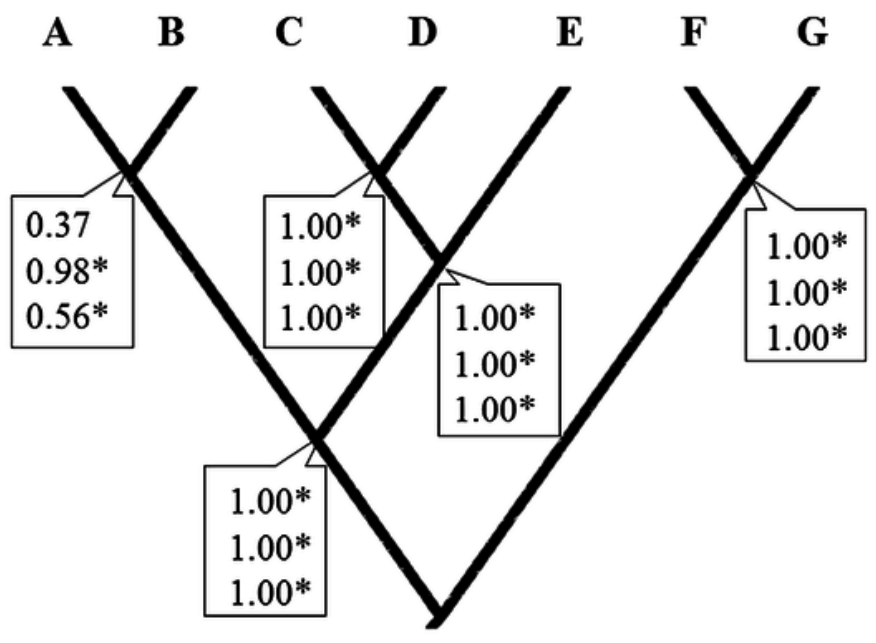

B 
Figure 3

Divergence times trees

Divergence times trees. (A) For multilocus dataset (Confidence interval values are inside parentheses), calibration points are shown in the time bar. (B) For ND2 dataset, using a sustitution rates of $2.5 \times 10^{-2}$ sustitutions/sites/lineages/Myr .

A

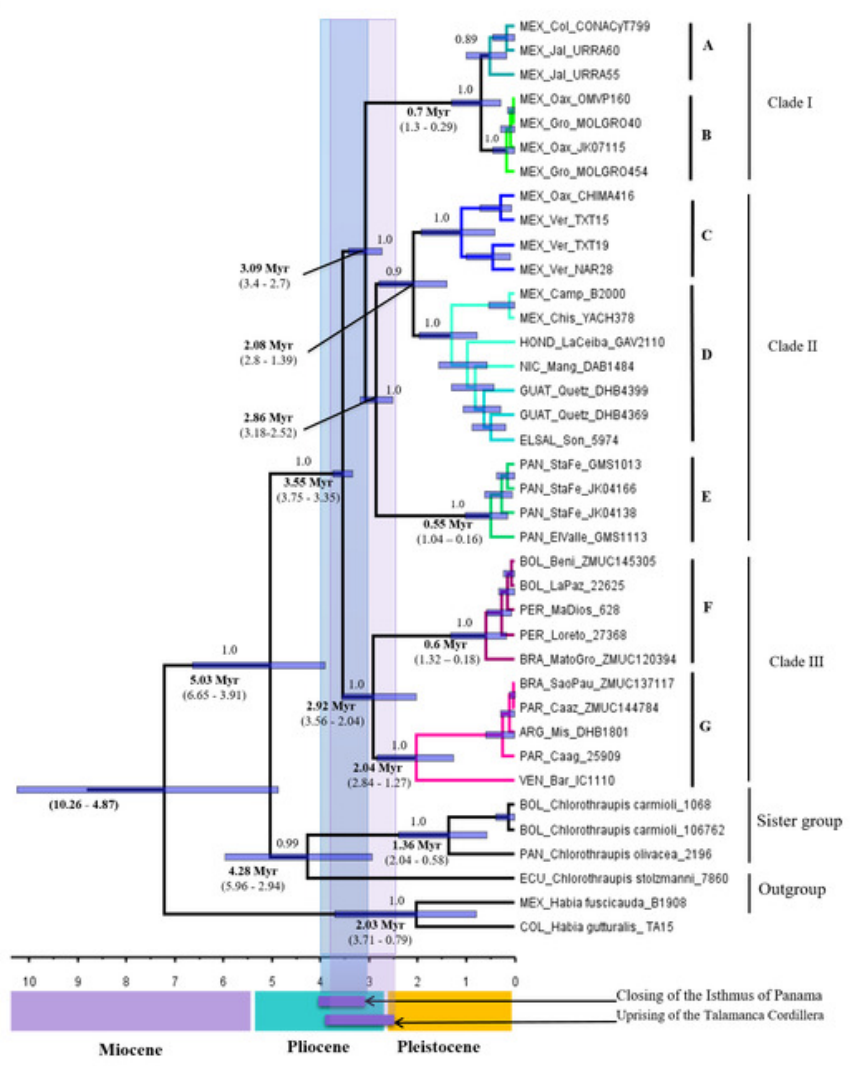

B

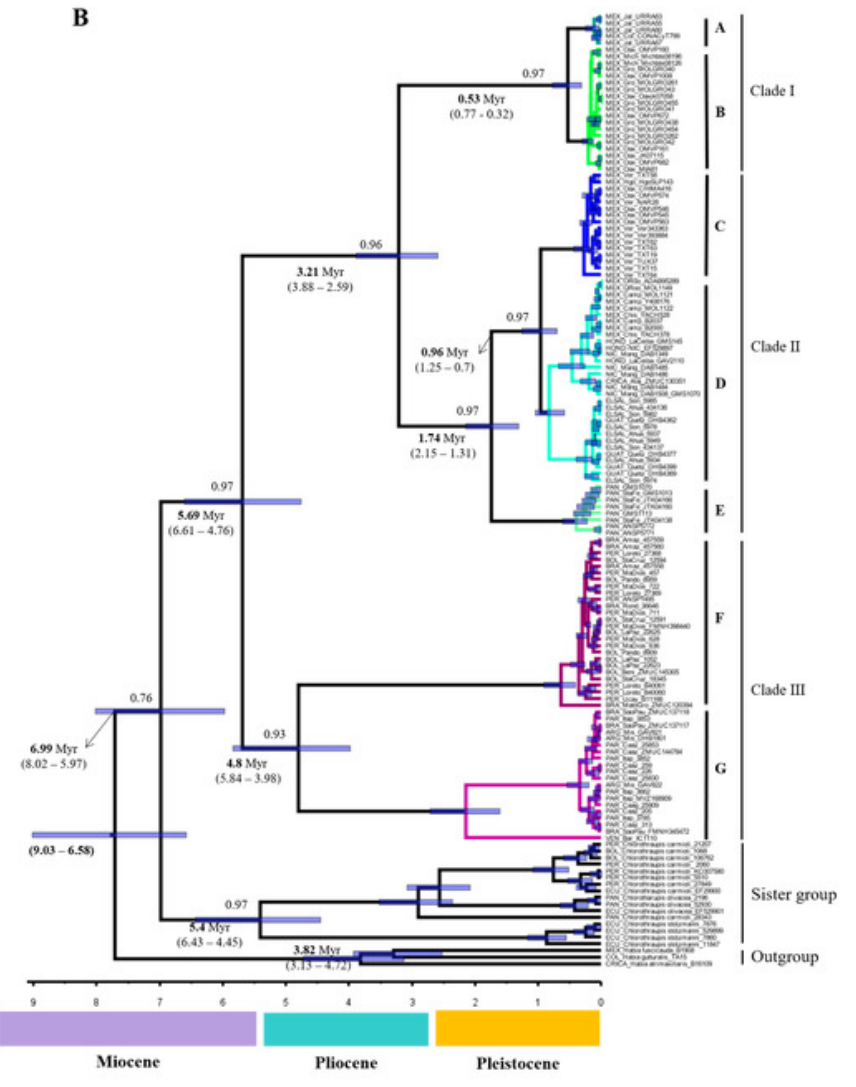




\section{Table $\mathbf{1}$ (on next page)}

Pairwise genetic $p$-distances 
1

\begin{tabular}{|c|c|c|c|c|c|c|c|}
\hline Phylogroups & $\mathbf{A}$ & B & $\mathrm{C}$ & D & $\mathbf{E}$ & $\mathbf{F}$ & G \\
\hline & \multicolumn{6}{|c|}{ mtDNA } & \\
\hline A & & & & & & & \\
\hline B & $0.7^{* *}$ & & & & & & \\
\hline C & $4.1^{*}$ & $4.4^{*}$ & & & & & \\
\hline D & $4.1^{*}$ & $4.5^{*}$ & $1.7^{* *}$ & & & & \\
\hline $\mathbf{E}$ & $5.1^{*}$ & $5.5^{*}$ & $2.7 * *$ & $2.4^{* *}$ & & & \\
\hline $\mathbf{F}$ & $6.4^{*}$ & $6.4^{*}$ & $5.7^{*}$ & $5.8^{*}$ & $6.6^{*}$ & & \\
\hline G & $6.8^{*}$ & $6.6^{*}$ & $5.7^{*}$ & $5.8^{*}$ & $6.7^{*}$ & $5.4^{*}$ & \\
\hline & & & & nDNA & & & \\
\hline A & & & & & & & \\
\hline B & $0.0^{* *}$ & & & & & & \\
\hline $\mathbf{C}$ & $0.1^{* *}$ & $0.1^{* *}$ & & & & & \\
\hline D & $0.1^{* *}$ & $0.1^{* *}$ & $0.1^{* *}$ & & & & \\
\hline $\mathbf{E}$ & $0.1^{* *}$ & $0.1^{* *}$ & $0.1^{* *}$ & $0.1 * *$ & & & \\
\hline $\mathbf{F}$ & $0.2^{* *}$ & $0.2^{* *}$ & $0.1 * *$ & $0.2 * *$ & $0.2 * *$ & & \\
\hline G & $0.6^{* *}$ & $0.6^{* *}$ & $0.5^{* *}$ & $0.6^{* *}$ & $0.6^{* *}$ & $0.4^{* *}$ & \\
\hline
\end{tabular}

$*<0.05$

$* *<0.005$

Corrected pairwise genetic p-distances between lineages of Habia rubica based on mitochondrial and nuclear markers. 
Table 2 (on next page)

Basic Information of mitochondrial and nuclear DNA 
1

\begin{tabular}{ccccccccc}
\hline Molecular marker & Samples & Sequences & $\begin{array}{c}\text { Bases } \\
\text { pair }\end{array}$ & $\begin{array}{c}\text { Monomorphic } \\
\text { sites }\end{array}$ & $\begin{array}{c}\text { Polymorphic } \\
\text { sites }\end{array}$ & $\begin{array}{c}\text { More than two } \\
\text { variants }\end{array}$ & $\begin{array}{c}\text { Gaps } \\
\text { Missing } \\
\text { data }\end{array}$ \\
\hline $\begin{array}{c}\text { Mitochondrial } \\
\text { DNA }\end{array}$ & 125 & 125 & 1041 & 782 & 259 & 229 & - \\
ACOI-I9 & & & & & & & \\
MUSK & 38 & 76 & 852 & 452 & 112 & 8 & 7 \\
FGB-I5 & 38 & 76 & 576 & 181 & 54 & 281 & 4 \\
ODC & 39 & 78 & 711 & 584 & 46 & 4 & 2 & 279 \\
Nuclear DNA & 37 & 74 & 2716 & 1733 & 268 & 17 & 8 \\
\hline
\end{tabular}

2

3 Relative amounts of information in the mitochondrial and nuclear DNA. 